\section{Case Reports in Oncology}

\title{
An Uncommon Recurrent Metastasis of Ovarian Immature Teratoma to the Small Bowel
}

\author{
Mohammad Al-hazaimeh ${ }^{a} \quad$ Mahmoud Jaradat $^{a}$ Mohammed El-Sadoni ${ }^{b}$ \\ Tamara Smadi $^{a} \quad$ Ruba Shannaq $^{c}$ Omar Bani Hani ${ }^{d} \quad$ Ahmad Alhesa $^{b}$ \\ Nisreen Abu Shahin ${ }^{b}$ Tareq Saleh ${ }^{\mathrm{e}}$ \\ aDepartment of General Surgery, Jordanian Royal Medical Services, Amman, Jordan; \\ ${ }^{b}$ Department of Pathology, Microbiology and Forensic Medicine, School of Medicine, \\ The University of Jordan, Amman, Jordan; 'Department of Pathology, King Hussein \\ Medical Center, Royal Medical Service, Amman, Jordan; ${ }^{\mathrm{d} D e p a r t m e n t}$ of Diagnostic \\ Radiology, Al-Bashir Hospital, Ministry of Health, Amman, Jordan; 'Department of Basic \\ Medical Sciences, Faculty of Medicine, The Hashemite University, Zarqa, Jordan
}

\author{
Keywords \\ Mature $\cdot$ Immature $\cdot$ Teratoma $\cdot$ Metastasis $\cdot$ Small bowel
}

\begin{abstract}
Immature ovarian teratomas are rare ovarian germ cell tumors associated with a variable potential of distant metastasis. The acquisition of mature phenotypes upon post-treatment recurrence of immature teratomas has been previously described. In this study, we report, for the first time, a rare case of a recurrent ovarian immature teratoma with mature deposits in the small bowel. An incidental pelvi-abdominal mass was identified in a 30-year-old pregnant patient during antenatal ultrasonography. The mass, which was resected through salpingooopherectomy, was histopathologically characterized as an immature teratoma of grade 2 and treated with 3 cycles of chemotherapy. After 3 years of completing treatment, the patient suffered from severe anemia which was investigated by capsule endoscopy that identified a bleeding source in the ileum. Imaging studies revealed an intrabdominal mass that was resected laparoscopically. The pathological assessment of the mass identified a submucosal/intramuscular teratoma with mature elements indicative of a recurrent metastasis of immature teratoma associated with post-chemotherapy retroconversion. The secondary mass was then managed with adjuvant chemotherapy.
\end{abstract}




\section{Introduction}

Teratomas of the ovaries are germ cell tumors that originate from all 3 germ cell layers containing different types of tissue such as hair, bone, and muscles [1]. Teratomas can be categorized into mature and immature, depending on the type of differentiation. Immature teratomas contain a variable amount of embryonal-type neuroectodermal tissues and are the second most common germ cell neoplasms of the ovaries and a cause for cystectomy and oophorectomy [2]. However, immature teratomas are still considered relatively rare, accounting for $<1 \%$ of all ovarian cancers [3]. While immature teratomas have more malignant nature than their mature counterparts, they still have a low potential to metastasize depending largely on their histological grade [4]. The most reported distant sites of blood-borne metastasis of immature teratomas include the liver, lungs, and brain [5]. Nevertheless, the spread of ovarian immature teratoma to the small bowel has not been described before. Moreover, recurrent immature teratomas are known to undergo pathological retroconversion to contain mature elements following chemotherapeutic treatment [5]. Here, we report a rare case of a small bowel lesion with mature teratoma features that developed 3 years following the resection and chemotherapeutic treatment of an immature ovarian teratoma in a 33-year-old female patient.

\section{Case Report/Case Presentation}

In May 2018, a 30-year-old female patient who was pregnant (G3P2) in her first trimester was found to have an incidental left ovarian cyst during a routine antenatal visit. Ultrasound imaging showed a $4 \times 4 \mathrm{~cm}$ solid mass in the left ovary. Magnetic resonance imaging (MRI) identified a large, well-defined heterogeneous pelvi-abdominal lesion epicentered on the left adnexa posterosuperior to the gravid uterus (shown in Fig. 1). The lesion exhibited predominant T2 hyperintense and predominant $\mathrm{T} 1$ hypointense with hyperintense areas within. On T1 fat-saturated images, the T1 hyperintense areas showed complete signal drop. The lesion measured about $12.5 \times 15.5 \times 13.5 \mathrm{~cm}$ in anteroposterior, width, and craniocaudal dimensions, respectively. Therefore, in July 2018, she underwent a left salpingo-oophorectomy. Pathological assessment of the resected mass revealed a mixture of mature tissue types including squamous epithelium, sebaceous glands, hair follicles, brain tissue, cartilage, smooth muscle, fibroadipose tissue, and respiratory epithelium (shown in Fig. 2a). Thorough examination, however, displayed multiple foci of admixed immature elements, mainly immature neuroepithelium. The cells in these foci appeared primitive, with scant cytoplasm, hyperchromatic nuclei, and frequent mitoses, with rosette, pseudorosette, and primitive tubule formation, which helped to differentiate the immature elements from mature brain tissue (shown in Fig. $2 \mathrm{a}$-inset). As the immature neuroepithelium was identified in $>1$ low power field in some slides, the ovarian mass was diagnosed as an immature teratoma of grade 2 (shown in Fig. 2a). The tumor was confined to the ovary without evidence of ovarian surface or fallopian tube involvement. Samples from pelvic lymph nodes and omentectomy were free of tumor and unremarkable. The histopathological stage was reported as pT1N0 (FIGO stage IA). Her postoperative serum levels of alpha-fetoprotein (AFP), human chorionic gonadotropin (hCG), and CA-125 levels were within the normal range (shown in Table 1).

Following the discussion of the treatment strategy by the onco-surgical multidisciplinary team, the patient received 3 cycles of adjuvant chemotherapy (namely: bleomycin, cisplatin, and vincristine), while she was still in her second trimester. At the last chemotherapy session, no residual tumor had been observed. In November 2018, she gave birth to a female baby through a cesarean section. Thereafter, the patient was followed-up by the oncology team and

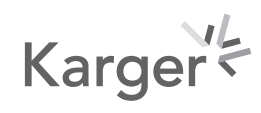




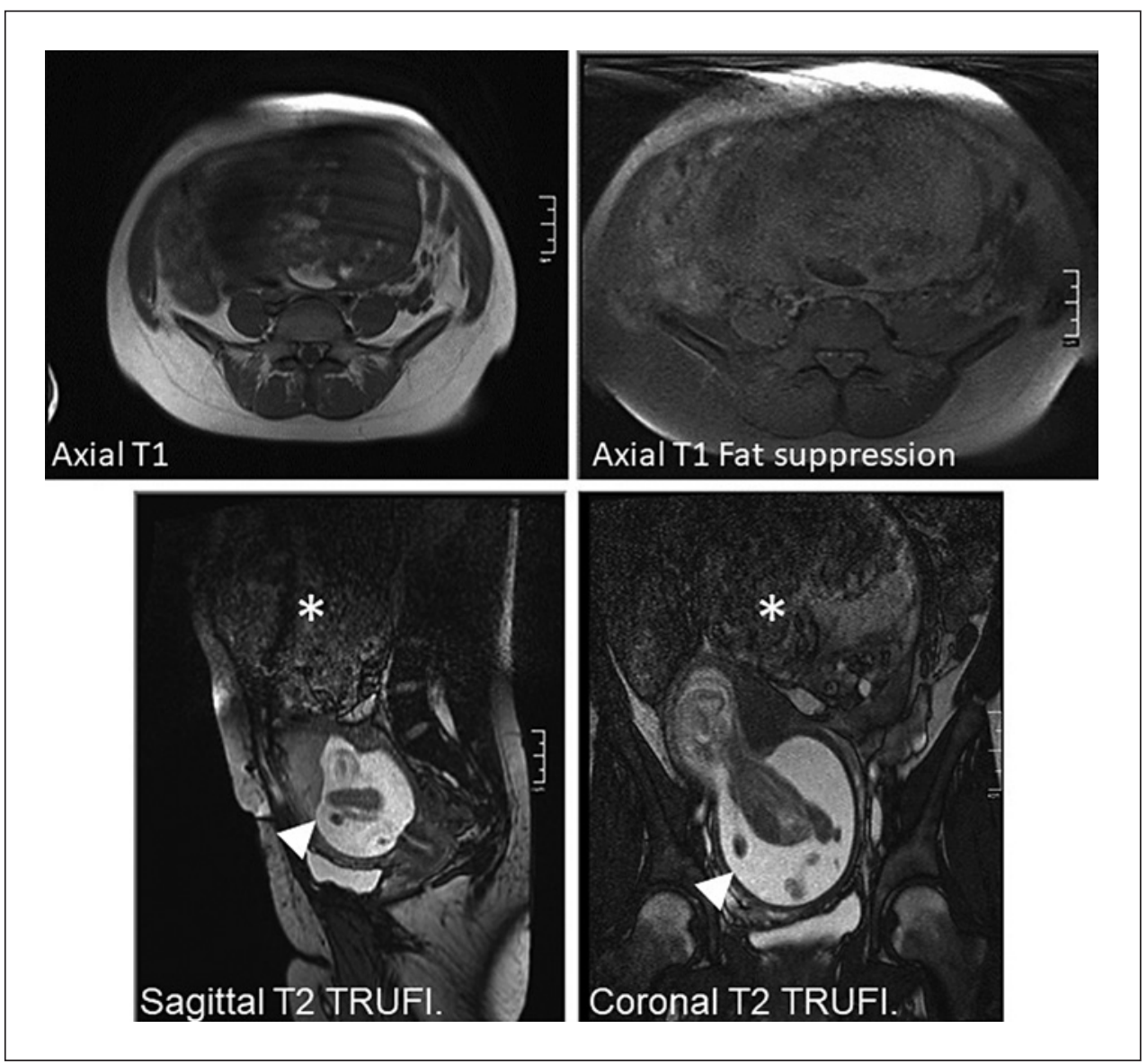

Fig. 1. MRI of the primary ovarian mass. Immature ovarian teratoma (asterisk). Gravid uterus (arrowhead). MRI, magnetic resonance imaging.

showed no signs or symptoms of recurrence until May 2020, when she started to complain of fatigue and dizziness. Upon physical examination of the abdomen, there were no masses or tenderness. Her blood tests showed evidence of iron deficiency anemia. Subsequently, the patient underwent upper and lower gastrointestinal endoscopy which failed to identify a source of bleeding that explains the cause of her anemia. During that time, she was admitted multiple times to the hospital for blood transfusion for the management of symptomatic anemia and was investigated again with upper endoscopy, push enteroscopy, and colonoscopy, all of which were inconclusive. In April 2021, she underwent a capsule video endoscopy for further investigation of potential sources of blood loss, which was successful in detecting a bleeding source in the terminal ileum. Contrast-enhanced abdominal computed tomography scan was ordered to detect possible intra-abdominal pathologies and revealed a non-enhancing lesion about $32 \times 37 \mathrm{~mm}$ at the proximal to mid-ileum region along with multiple lymph node enlargements. As a result, she underwent laparoscopic small bowel resection for the mass which was located $60 \mathrm{~cm}$ proximal to the ileocecal valve. Her postoperative histopathological report showed a solid and cystic mural and submucosal mass. Microscopic examination of the mass revealed a tumor with a mixture of mature tissues originating from all germ cell layers. Despite thorough exploration of the mass, surprisingly, no immature or malignant components were identified. The histopathological report suggested the diagnosis of a mature cystic teratoma (shown in Fig. 2b). Such findings are thought to denote conversion of deposits of 


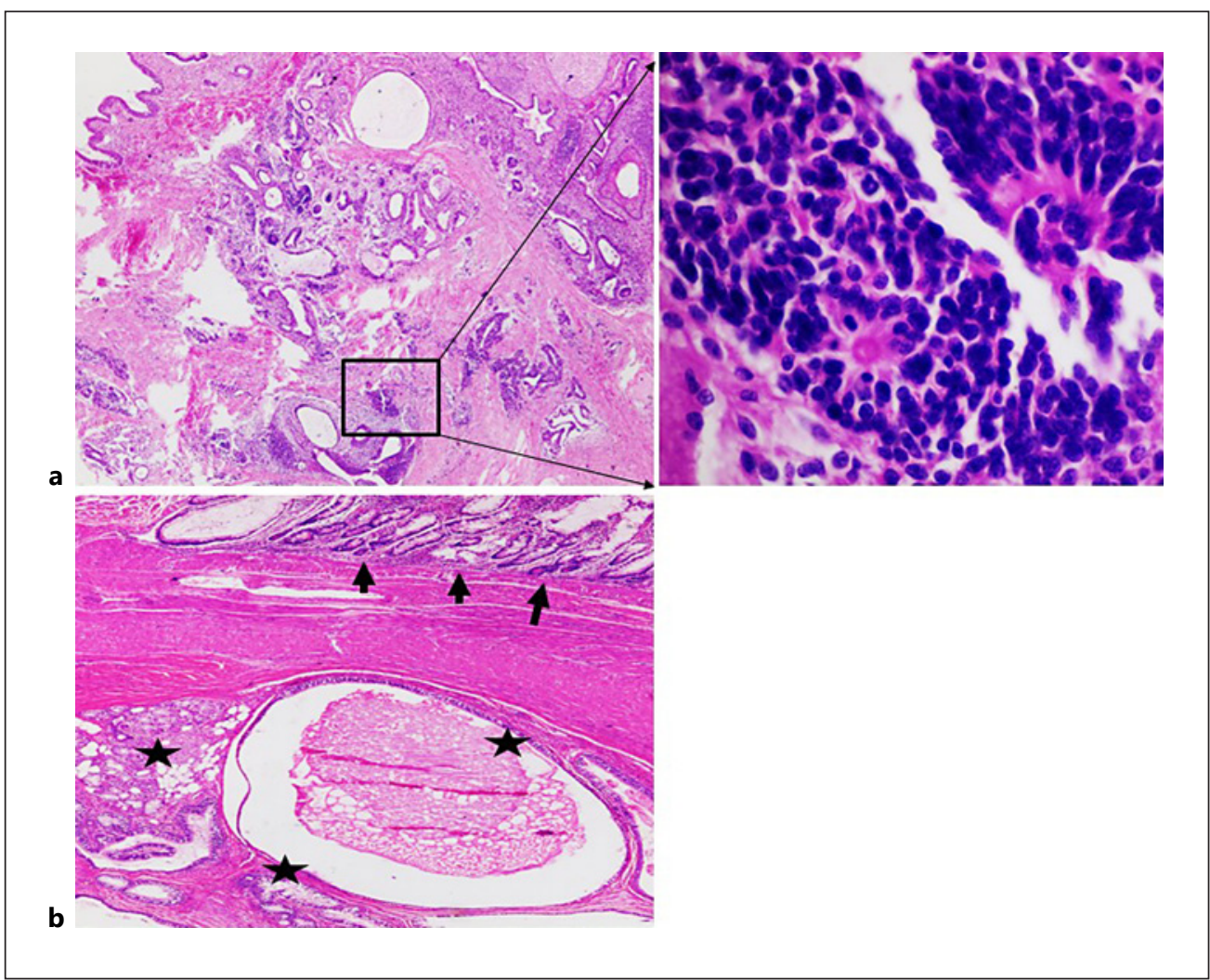

Fig. 2. Microscopic images from the ovarian mass and the intestinal mass. a Ovarian tumor with a mixture of mature and immature neuroepithelial elements (magnified in inset). b The intestinal mass identified as mature teratoma (stars) underneath the unremarkable intestinal mucosa (arrows). Hematoxylin and eosin stain; original magnifications, $\times 40, \times 400$, and. $\times 100$, respectively.

immature teratoma into mature teratoma because of chemotherapy (chemotherapeutic retroconversion) [5-7].

Accordingly, and following a discussion of management approaches by a multidisciplinary team, the patient was commenced on a second-line adjuvant chemotherapy of etoposide, ifosfamide, and cisplatin. Her chemotherapy treatment was complicated by neutropenia and urinary tract infection in August 2021, for which she was admitted to the hospital and treated conservatively with intravenous antibiotics. A marked decrease in the levels of AFP and hCG was recorded (as shown in Table 1).

\section{Discussion/Conclusion}

Despite comprising only $1 \%$ of total ovarian malignancies, immature teratoma account for $20 \%$ ovarian germ cell cancers. Microscopically, immature teratomas are characterized by the presence of microscopic foci of immature neuroepithelial tissue and rapid growth marked by capsular invasion [8]. Treatment for immature ovarian teratomas is grade dependent; while patients with stage Ia grade 1 immature teratomas are often treated with surgical resection (mainly in the form of salpingo-oophorectomy) of the mass due to favorable prognosis, grade 2 or 3 , or advanced stage teratomas require operative intervention combined with chemotherapy [4]. Unfortunately, immature teratomas can be complicated with relapse [9]. 
Table 1. Serum levels of several biomarkers associated with immature teratoma

\begin{tabular}{lllllll}
\hline Date & $\begin{array}{l}\text { Timely phase of } \\
\text { treatment }\end{array}$ & AFP, ng/mL & hCG, mIU/mL & CA-125, U/mL & CA-19-9, U/mL & CEA, ng/mL \\
\hline 11 Dec 2018 & $\begin{array}{l}\text { Postoperative } \\
\text { (primary tumor) }\end{array}$ & 4.16 & $<1.00$ & 17.12 & $N / A$ & $N / A$ \\
29 Apr 2019 & Follow-up & 5.81 & 0.18 & $N / A$ & $N / A$ & $N / A$ \\
3 Jan 2019 & Follow-up & 1.95 & $N / A$ & 16.50 & $N / A$ & $N / A$ \\
29 Jul 2019 & Follow-up & 1.58 & 0.71 & $N / A$ & $N / A$ & $N / A$ \\
2 Feb 2020 & Follow-up & 1.17 & 0.23 & $N / A$ & $N / A$ & $N / A$ \\
10 Aug 2020 & $\begin{array}{l}\text { Postoperative } \\
\text { (secondary tumor) }\end{array}$ & 1.50 & 0.00 & 10.50 & 4.94 & $<0.10$ \\
\hline
\end{tabular}

Table shows serum levels of AFP, hCG, CA-125, CA-19-9, and CEA over the course of treatment. Primary tumor (ovarian immature teratoma) was resected through salpingo-oophorectomy, while the secondary tumor (small bowel mature teratoma) was removed through laparoscopic segmental small bowel resection.

AFP, alpha-fetoprotein; hCG, human chorionic gonadotropin; CEA, carcinoembryonic antigen.

Risk for relapse of immature teratoma depends largely on the histological grading based on the extent of immature elements within the tumor [9]. Herein, we describe a case of relapse of a grade 2 immature ovarian teratoma in a 33-year-old female patient which occurred within 3 years of treatment. This observation is in consistence with previous findings indicating that the recurrence of immature teratomas often occurs within 2-3 years after treatment [10] but represents a somewhat rare incident since only $~ 4 \%$ of grade 2 immature teratomas of the ovary are complicated by relapse [9].

Hematogenous metastasis of ovarian immature teratomas is infrequent. The majority of distant metastases were identified in the liver $[5,11]$. Other intrabdominal metastatic foci have been reported to occur in the retroperitoneum [12,13] and omentum [14]. Supradiaphragmatic metastasis to the thoracic cavity [15] or brain has been also described [5]. Rare sites of distant spread include soft tissue of the thigh [16] and abdominal wall musculature [17]. Interestingly, the nature of the metastatic lesions was markedly consistent of mature tissue, with absence of immature elements, a phenomenon first described by Logothetis et al. [18] and referred to as growing teratoma syndrome (GTS). Additionally, these recurrent teratoma lesions are often detected following or during chemotherapeutic treatment which is referred to as chemotherapeutic retroconversion (first characterized by DiSaia et al. [19]). Despite being described separately, accumulative reports highly indicate that both terms are likely to refer to the same process [6]. However, a main difference is that with retroconversion, the arousal of metastatic disease is not associated with mass expansion which seems to characterize GTS [20]. In this case presentation, we report the development of recurrent metastasis of immature teratoma in the small bowel (ileum) associated with retroconversion to mature teratoma 3 years following the completion of chemotherapy. While metastatic spread to parts of the omentum and retroperitoneal space was documented before, to our knowledge, this is the first time where metastatic involvement of the small bowel is reported. In this report, the secondary lesion comprised solid and cystic mural and submucosal mass within the wall of the ileum containing no immature tissue elements.

The pathogenetic basis of this phenomenon is not well understood. Although conversion to mature phenotypes has been described regardless of the chemotherapeutic drug combination used for treatment, exposure to chemotherapy appears to be the key step. Kataria et al. [20] reviewed potential mechanistic explanations for retroconversion as suggested in the literature. Of those, the hypothesis that chemotherapy is capable of suppressing the 
growth of immature components selectively allowing for mature tissue to grow over time is most plausible [21]. This suggests that metastatic deposits are likely to consist of immature tissue early on which matures gradually following exposure to chemotherapy. Unfortunately, it is challenging to confirm this proposition due to the lack of reliable preclinical models. Lastly, normalization of serum levels of AFP, hCG, CA-125, and AFP is a frequent observation in GTS; however, in the current case, the patient maintained normal ranges of these biomarkers despite a notable reduction in their serum levels following the surgical removal of the ovarian tumor and treatment with chemotherapy.

In conclusion, this case report provides additional evidence on the recurrent metastasis of ovarian immature teratoma and conversion into mature lesion following exposure to chemotherapy but in the small bowel, a previously unreported metastatic destination. The importance of reporting these cases, despite being rare, is to direct attention of clinicians and pathologists towards the possibility of unusual clinicopathological presentation of ovarian germ cell line tumors, especially that early intervention is associated with better outcomes.

\section{Acknowledgment}

The authors would like to thank the Deanship of Scientific Research at The Hashemite University.

\section{Statement of Ethics}

All research and clinical activities reported in this work were conducted ethically in accordance with the World Medical Association Declaration of Helsinki. This study's protocol was reviewed and approved by the IRB committee at the Royal Jordanian Medical Services approval number (11/2021). A written informed consent was obtained from the patient allowing for the publication of the details of her medical case and accompanying investigational results.

\section{Conflict of Interest Statement}

The authors have no conflict of interest to declare.

\section{Funding Sources}

This research effort received no funding.

\section{Author Contributions}

M.A. conducted evaluation of the case's clinical history and physical findings, wrote the case scenario, and obtained ethical approval. M.J. and T.Z. revised and edited the clinical description of the case. M.E.-S. and A.A. collected patient samples and performed histochemical staining. R.S. and N.A.S. conducted pathological evaluation of the primary and secondary tumor samples. O.B.H. provided detailed radiological assessment. T.S. directed the research activities, wrote and revised the manuscript, and supervised the work.

\section{Karger'}




\section{Data Availability Statement}

All data generated or analyzed during this study are included in this article. Other information is not publicly available due to the possibility of compromising the privacy of the research participant but is available from the corresponding author T.S.

\section{References}

1 Tavassoli FA, Devilee P. Pathology and genetics of tumours of the breast and female genital organs. Lyon, France: IARC Publications; 2002.

2 Vicus D, Beiner ME, Clarke B, Klachook S, Le LW, Laframboise S, et al. Ovarian immature teratoma: treatment and outcome in a single institutional cohort. Gynecol Oncol. 2011;123:50-3.

3 Debuquoy C, Romeo C, Vanacker H, Ray-Coquard I. Rare ovarian tumors: an update on diagnosis and treatment. Int J Gynecol Cancer. 2020;30:879-87.

4 O'Connor DM, Norris HJ. The influence of grade on the outcome of stage I ovarian immature (malignant) teratomas and the reproducibility of grading. Int J Gynecol Pathol. 1994;13:283-9.

5 Kurata A, Hirano K, Nagane M, Fujioka Y. Immature teratoma of the ovary with distant metastases: favorable prognosis and insights into chemotherapeutic retroconversion. Int J Gynecol Pathol. 2010;29:438-44.

6 Amsalem H, Nadjari M, Prus D, Hiller N, Benshushan A. Growing teratoma syndrome vs. chemotherapeutic retroconversion: case report and review of the literature. Gynecol Oncol. 2004;92:357-60.

7 Lee YL, Lai CR, Yen MS. Recurrent ovarian mixed germ cell tumor with unusual malignant transformation: a case report. J Ovarian Res. 2019;12:2-6.

8 Outwater EK, Siegelman ES, Hunt JL. Ovarian teratomas: tumor types and imaging characteristics. Radiographics. 2001;21:475-90.

9 Pashanka F, Hale JP, Dang H, Krailo M, Brady WE, Rodriguez-Galindo C, et al. Is adjuvant chemotherapy indicated in ovarian immature teratomas? A combined data analysis from the Malignant Germ Cell Tumor International Collaborative. Cancer. 2016;122:230-7.

10 Murugaesu N, Schmid P, Dancey G, Agarwal R, Holden L, McNeish I, et al. Malignant ovarian germ cell tumors: identification of novel prognostic markers and long-term outcome after multimodality treatment. J Clin Oncol. 2006;24:4862-6.

11 Caldas C, Sitzmann J, Trimble CL, Mcguire WP. Synchronous mature teratomas of the ovary and liver: a case presenting 11 years following chemotherapy for immature teratoma. Gynecol Oncol. 1992;47:385-90.

12 Li S, Liu Z, Dong C, Long F, Liu Q, Sun D, et al. Growing teratoma syndrome secondary to ovarian giant immature teratoma in an adolescent girl: a case report and literature review. Medicine. 2016;95:e2647.

13 Block M, Gilbert E, Davis C. Metastatic neuroblastoma arising in an ovarian teratoma with long-term survival. Case report and review of the literature. Cancer. 1984;54(3):590-5.

14 Frazer JL, Hook CE, Addley HC, Jackson CR, Latimer JA, Nicholson JC, et al. Recurrent ovarian immature teratoma in a 12-year-old girl: implications for management. Gynecol Oncol. 2019;154:259-65.

15 Nogales FF, Favara BE, Major FJ, Silverberg SG. Immature teratoma of the ovary with a neural component ("solid" teratoma). A clinicopathologic study of 20 cases. Hum Pathol. 1976;7:625-42.

16 Byun JC, Choi IJ, Han MS, Lee SC, Roh MS, Cha MS. Soft tissue metastasis of an immature teratoma of the ovary. J Obstet Gynaecol Res. 2011;37:1689-93.

17 Swartjes JM, de Blok S, Blaauwgeers JLG. Abdominal wall metastases after surgical resection of an immature teratoma of the ovary. Eur J Obstet Gynecol Reprod Biol. 1997;74:41-3.

18 Logothetis CJ, Samuels ML, Trindade A, Johnson DE. The Growing teratoma syndrome. Cancer. 1982;50(8): 1629-35.

19 DiSaia PJ, Saltz A, Kagan AR, Morrow CP. Chemotherapeutic retroconversion of immature teratoma of the ovary. Obstet Gynecol. 1977;49:346-50.

20 Kataria SP, Varshney AN, Nagar M, Mandal AK, Jha V. Growing teratoma syndrome. Indian J Surg Oncol. 2017; 8:46.

21 André F, Fizazi K, Culine S, Droz J, Taupin P, Lhommé C, et al. The growing teratoma syndrome: results of therapy and long-term follow-up of 33 patients. Eur J Cancer. 2000;36:1389-94. 